\title{
Intensive Care Medicine: Where We Are and Where We Want To Go?
}

\author{
R.P. Moreno and A. Rhodes
}

\section{Introduction}

Intensive care medicine can be defined as the science and art of detecting and managing patients with impending or established critical illness, in order to prevent further deterioration and revert the disease process or its consequences, so as to achieve the best possible outcomes.

\section{Evolution of Intensive Care}

Florence Nightingale was best known as 'The Lady with the Lamp' for her habit of making rounds at night to tend to injured soldiers during the Crimean war (October 1853 - February 1856). She recognized that some patients needed more frequent and careful monitoring than others and as a consequence started to place these patients closer to the nursing station [1]. It could be argued that this was the beginnings of the specialty of intensive care.

Similar insights also began to emerge in other parts of the world [2]. As a consequence of the 1952 Copenhagen poliomyelitis epidemic [3, 4], hospitals started to create the first areas specifically designed and adapted to provide intense support for failing organ systems. The introduction of this new branch of medical science (both physiological and technological), quickly required the development of a new setting for these skills, and the subsequent creation of a designated area in the hospital, known today as the intensive care unit (ICU). Subsequent to this, many different individuals, including Vladimir Negovsky, Peter Safar, Max Harry Weil amongst others, created the science of reanimatology [5, 6]. These pioneers, together with subsequent generations of clinicians and nurses, have continued to develop new knowledge and skills as well as the technology required to transform this diverse series of competencies into an integrated package of care, now known as the art and science of intensive care medicine [7].

\section{Why We Need Intensive Care Units}

It is the concentration of the skills, expertise and resources (both human and technical) together in one designated area that makes an ICU. This concentration enables optimal care and management of patients to be provided. There is reasonable evidence now that demonstrates that the care of critically ill patients by intensivists and critical care trained nurses can improve many patient related out- 
comes as well as using the available resources more efficiently. These improved outcomes include a reduced rate of nosocomial infections, decreased complications, reduced length of ICU stays, and decreased mortality. If these resources are not concentrated, rather spread evenly throughout the hospital, with a couple of beds on each ward, then this 'improvement by quantity' is reduced with a less efficient use of resources and a decreased level of care being delivered to the patients.

\section{The ICU of Today}

The ICU is critically involved with many areas and specialties within the hospital. The location chosen for the ICU, however, commonly reflects the need for geographic proximity to the more acute areas, such as the emergency department and operating rooms. In addition, there needs to be some thought towards colocation with diagnostic facilities such as the availability of a computed tomography (CT) scanner and the availability of a 24-hour seven-day-a-week functioning laboratory. Most ICUs today have facilities and equipment located within the unit to perform the immediate analysis of arterial blood gas samples and some basic biochemistry and hematology tests. Since most situations in intensive care medicine are critically time-dependent, the successful provision of care is reliant on good relationships and communication between the ICU and the other services and departments of the hospital. This is also required to optimize the timing of admission and discharge of patients to, and from, the ICU.

The discharge process is especially important for a number of reasons [8]. A higher level of vigilance of recently discharged patients is necessary to identify and prevent any subsequent deterioration early. Failure to do so can compromise the patient and reverse any benefits that the ICU initially provided $[9,10]$. Another risk to the recovering intensive care patient is inappropriate early discharge to the ward, due to lack of beds or time constraints [11], and although this is controversial $[12,13]$, it has been demonstrated that there is scope for improvement [14]. This need for an effective interface between the ICU and the other departments of the hospital has not only physical and architectural implications: it also has a crucial impact on human resource factors, both outside and inside the ICU. These include stress management, professionalism in facing and coping with rotating working patterns [15], and fatigue [16, 17].

\section{Interfacing the ICU}

Today, a significant number of ICUs in Europe (and also in Australia and New Zealand) are directed by a full-time, fully trained intensivist, leading a multi-professional team of experts in the field, able to provide all interventions potentially required by the patient 24 hours a day, 7 days a week. These professionals are also more and more involved in the management of unstable patients outside the ICU [18-21] in a movement called by Ken Hillman "critical care without walls" [21]. In the United States, the situation is slightly different, with a significant number of ICUs still using the so-called open model [22]. In this system, care and therapy are often supervised by nurses and younger physicians (with occasional mandatory or optional consultation with an intensive care professional), but under the 
direction and orientation of a primary physician, paradoxically a system that the literature suggests provides less effective care [23].

This need for full-time, fully trained teams, with very intensive physician-topatient and nurse-to-patient ratios, transformed the ICU into a very expensive and often scarce resource. The obvious implication of this is that it is vital to ensure that capacity is reserved for only those most likely to benefit from it. There is, therefore, a significant responsibility for the intensivist to triage patients appropriately so that those most likely to benefit are admitted, and those less likely to, are not. Often, however, the situation is more complex. ICU is but one part of the patient journey that takes in many differing parts of the hospital together with multiple professional consultations and interventions. ICU admission criteria must, therefore, be able to cope with not just the sick emergency patient but often also the elective patient whose admission has been planned even before hospital admission.

\section{Triage of Admissions and Discharges}

The development of admission (and discharge) criteria is a very complex issue, full of ethical implications, both to the patient and to society. Being a potentially life-saving asset, an ICU bed is also a scarce and costly resource that should be used in the most cost-effective way. Consequently, all possible expertise should be used when deciding whether a certain patient should, or should not, be admitted to an ICU, a decision that is notoriously difficult to get right with any precision [24]. Usually, several objective and subjective factors - both ICU related and patient-related - have an impact on this decision, such as the number of beds available, the admission diagnosis, the severity of illness, age and operative status [25]. Several proposals for standards for ICU admission have been proposed, with the most well-known being those from the Society of Critical Care Medicine [26] (Box 1).

In a recent multicenter study in France, Maité Garrouste-Orgeas et al. [27] demonstrated that the decision to deny ICU admission to a certain patient was common (23.8\%), explained by the patient being too well to benefit (55.4\%), too sick to benefit $(37.2 \%)$, the unit too busy $(6.5 \%)$, and/or refusal by the family

Box 1. Scheme of priorities to assess triage decisions [26]

- Priority 1 assigned to patients who are critically ill, unstable, in need of intensive treatment and monitoring that cannot be provided outside of the ICU. No limits are generally placed on the extent of the therapy that these patients can receive

- Priority 2 assigned to patients who require intensive monitoring, and may potentially need immediate intervention. This category includes patients who are at risk for intubation and invasive mechanical ventilation. No therapeutic limits are generally placed for these patients

- Priority 3 assigned to patients with underlying disease and/or acute illness with a reduced likelihood of recovery. Due to their long-term outcome, they may receive intensive treatment to relieve acute illness, but limits on therapeutic efforts may be set

- Priority 4 assigned to those who are generally not appropriate for ICU admission, either because they are 'too well to benefit' or 'too sick to benefit'. This level also includes those patients who have the capacity to make decisions and who decide to refuse aggressive interventions, although still require 'comfort' care at a level not deliverable on a normal ward setting. 
$(0.7 \%)$. The same authors demonstrated in multivariate analysis that the two patient-related factors more strongly associated with ICU refusal were dependency and metastatic cancer and that the most important organizational factors were the unit being full, the specific center, phone rather than face to face referral, and daytime admissions (odds ratio [OR] 0.52; $95 \%$ confidence interval [CI] $0.32-0.84)$ [27].

Given the uncertainty of all these decisions, several authors in recent years have proposed that for patients with very severe disease, especially those with cancer, that a so-called 'ICU trial' should be offered; in other words, patients are admitted and fully treated for a limited period of time and then re-assessed for the continuation of life-sustaining therapy $[28,29]$. If the patient is not benefiting from the ICU care, then appropriate decisions with regards end-of-life-care should be made, according to the state of the art, the law, and religious preferences, a process quite heterogeneous in different cultures [30-33].

For situations where the demand for intensive care could largely exceed supply in a short period of time, as happened during the epidemic of severe acute respiratory syndrome (SARS) in Hong-Kong [34] and in Toronto [35], or in certain places of the world during the recent influenza A (H1N1) virus pandemic $[36,37]$, contingency plans should exist in anticipation for both the need to increase the capacity of intensive care services and also for triage of patients who could benefit more from ICU admission, ideally based on objective and pre-defined criteria $[38,39]$.

As a consequence of all these factors, during their ICU stay all patients must be continuously evaluated for the need to remain in the ICU. According to consensus definitions, a discharge decision should be taken "when a patient's physiologic status has stabilized and the need for ICU monitoring and care is no longer necessary" [26]. However, this issue is more complex than it seems at first glance. Since the 1980s, many published outcome studies have presented data on vital status at ICU discharge and also at hospital discharge. Consequently, it has become clear that a significant number of patients either deteriorated or died following ICU discharge but before leaving the hospital (the so-called post-ICU mortality or occult mortality). Several published studies have raised attention to the magnitude of this phenomenon, which can be as high as $36.7 \%$ of all deaths [40]. Some patients deteriorate and then need to be re-admitted to the ICU often soon after ICU discharge [41-44], again a common phenomenon carrying a large associated mortality [45].

\section{Where are we Going and Where do we Want to Go?}

Our speciality has sustained a continuous growth in recent years. In the early 2000s, it was estimated that intensive care beds represented $13.4 \%$ of all hospital beds in the USA, costing upwards of $\$ 55.5$ billion, accounting for $13.3 \%$ of all hospital costs and $0.56 \%$ of the gross domestic product (GDP). In the last few years, the panorama has changed [46], with the number of intensive care beds, days (as a percentage of the total hospital days) and occupancy rates continuing to increase. Also, the costs per day of intensive care medicine have increased by $30.4 \%$ with a corresponding increase in the annual costs associated with this specialty of $44.2 \%$; in 2005 , this represented $13.4 \%$ of hospital costs, $4.1 \%$ of national health expenditure, and $0.66 \%$ of the GDP, If we add to this number 
other costs incurred by caring for patients with critical illness the overall number accounts for $1 \%$ of the GDP in the USA. In Europe, despite the fact that the heterogeneity is much greater across countries [47] or even inside the same country [48], the mean costs per intensive care bed per year could be as low as 30,990 euro or as high as between 225,000 to 471,330 euro in the UK (depending on the level of care) [48], similar to those in Germany [49]. Despite the fact that these numbers are consistently lower than the numbers presented in the USA, pressure on the economy remains an issue.

This panorama is likely to change, due to the increasing age of the population, with the increasing prevalence of comorbid diseases, together with significant advances in medical science. These factors when combined result in the application of more complex and costly procedures to an increasing fragile population, in which complications will have greater consequences due to the increasingly narrow cost-effective margin of a significant number of interventions. As a direct consequence of these changes, there has been a shift from the almost exclusive presentation in medical conferences and medical journals of new devices and drugs to an increasing discussion of topics that 15 years ago would not have been accepted in a large major conference, or would rarely have been heard. Examples are the increasing efforts put on patient safety [50-52], detection and prevention of adverse events [53-56], and cultural changes regarding patient safety and error management [57-61]. A major example of these changes in priorities and culture was the signature by more than 80 Scientific Societies, industry representatives and patient representatives of the Declaration of Vienna, during the last Annual Congress of the European Society of Intensive Care Medicine, a public call for attention and action to these issues [51]. This declaration was just a first step in an ongoing-process that includes the public presentation of a revised version of the structural norms for European ICUs [62] and the revision of mandatory indicators for ICU evaluation. Benchmarking and other methods of comparative evaluation of the effectiveness and the cost-effectiveness of ICUs will have a growing impact in the decisions made by purchasers of intensive care $[63,64]$

In the future, maximization of the volume-outcome relationship will certainly lead to the fusion (or the closure) of small ICUs [65-69] and to the re-arrangement of existing ICUs and services into large networks, trained and evaluated for organizational performance and not just for clinical performance [70]. New drugs and devices will be subjected to ever greater scrutiny before utilization, with the quality of the trials in which they proved their efficacy (and cost-efficacy) being more highly scrutinized for adverse events than previously, and clearly separating practice guidelines and clinical orientations from industry campaigns [71, 72]. We will certainly have new tools and devices, new drugs and interventions [73], but we cannot just sit and wait for a magic bullet to appear, we must be proactive in applying existing (and new) interventions to decrease mortality [74], which translate evidence into practice [75].

This optimization in the use of resources will allow us to develop better and earlier triage criteria and a more extensive use of ICU trials [28]. As the utilization of critical care expands, we will need to be increasingly conscientious that our efforts are being applied only to those patients most likely to benefit from them. End-of-life practices must, therefore, be incorporated into the assessment of quality $[30,76]$. For this to be fair, clear, honest and transparent these issues have to be better and more openly discussed with the patients and their families [77-79]. This debate must start before admission to intensive care with discus- 
sion and re-education that resets the expectations, desires and perceptions of the general public to allow for more rationale decision making and an assurance that these therapies are directed only to those most likely to benefit.

The education and training of the next generation of intensivists [80-82], needs to be re-evaluated. In the USA, it is probable that the pendulum has already swung beyond the point where the equilibrium between the need for these specialists and the ability to provide them can be restored just by training alone. This will inevitably result in increased outsourcing of several medical interventions to other professional groups, for instance the increase in the use of physicians-assistants and nursing practitioners. New technologies such as tele-ICU [83] can help solve this problem but their effectiveness has not yet been demonstrated in a convincing fashion, as recently shown $[84,85]$.

Europe, the home country of the closed ICU model and of the fully trained, fully dedicated intensivist, will make an effort to meet the increasing demand with more intensivists, shifting education and training programs from timebased to competency-based curricula $[86,87]$, such the CoBaTrICE (Competency Based Training program in Intensive Care Medicine for Europe) collaboration [88], and by an increased use of simulation for critical situations [89, 90]. This will not be an easy process. It will need a change in our perception of teaching and the skills of our teachers [91, 92], but it can and should be done.

\section{References}

1. Nightingale F (1863) Notes on Hospitals, 3rd ed. Longman, Green, Longman, Roberts, and Green, London 1863

2. Takala J (2007) Organisation of intensive care. In: Kuhlen R, Moreno R, Ranieri M, Rhodes A (eds) 25 Years of Progress and Innovation in Intensive Care Medicine. Medizinisch Wissenschaftliche Verlagsgesellschaft, Berlin, pp 343-350

3. Lassen HCA (1953) A preliminary report on the 1952 epidemic of poliomyelitis in Copenhagen with special reference to the treatment of acute respiratory insufficiency. Lancet 1: $37-41$

4. Ibsen B (1954) The anaesthetist's viewpoint on the treatment of respiratory complications in poliomyelitis during the epidemic in Copenhagen, 1952. Proc R Soc Med 47: 72-74

5. Negovsky VA (1961) Some physiopathologic regularities in the process of dying and resuscitation. Circ Metab Cerveau 23: $452-457$

6. Negovsky VA (1986) Essays on Reanimatology. MIR Publishers, Moscow

7. Moreno RP, Rhodes A (2010) Intensive Care: a specialty coming to life $376: 1275-1276$

8. Moreno R, Miranda DR, Matos R, Fevereiro T (2001) Mortality after discharge from intensive care: the impact of organ system failure and nursing workload use at discharge. Intensive Care Med 27: 999-1004

9. Moreno R, Agthé D (1999) ICU discharge decision-making: are we able to decrease postICU mortality? Intensive Care Med 25: 1035-1036

10. Iapichino G, Morabito A, Mistraletti G, Ferla L, Radrizzani D, Miranda RR (2003) Determinants of post-intensive care mortality in high-level treated critically ill patients. Intensive Care Med 29: 1751-1756

11. Goldfrad C, Rowan K (2000) Consequences of discharges from intensive care at night. Lancet 355: $1138-1142$

12. Bell CM, Redelmaier DA (2001) Mortality among patients admitted to hospitals on weekends as compared with weekdays. N Engl J Med 345: 663-668

13. Arabi Y, Alshimemeri A, Taher S (2006) Weekend and weeknight admissions have the same outcome of weekday admissions to an intensive care unit with onsite intensivist coverage. Crit Care Med 34: 605-611

14. Perren A, Conte P, De Bitonti N, Limoni C, Merlani P (2008) From the ICU to the ward: 
cross-checking of the physician's transfer report by intensive care nurses. Intensive Care Med 34: 2054-2061

15. Harrocks N, Pounder R (2006) Working the night shift: preparation, survival and recovery - a guide for junior doctors. Clin Med 6: 61-67

16. West CP, Tan AD, Habermann TM, Sloan JA, Shanafelt TD (2009) Association of Resident Fatigue and Distress With Perceived Medical Errors. JAMA 302: 1294-1300

17. Gaba DM, Howard SK (2002) Fatigue among clinicians and the safety of patients. N Engl J Med 347: 1249-1255

18. Hourihan F, Bishop GF, Hillman KM, Daffurn K, Lee AJ (1995) The medical emergency team: a new strategy to identify and intervene in high-risk patients. Clin Intensive Care 6: $269-272$

19. Lee AJ, Bishop GF, Hillman KM, Daffurn K (1995) The medical emergency team. Anaesth Intensive Care Med 23: 183-186

20. Hillman K, Parr M, Flabouris A, Bishop G, Stewart A (2001) Redefining in-hospital resuscitation: the concept of the medical emergency team. Resuscitation 48: 105-110

21. Hillman K (2002) Critical care without walls. Curr Opin Crit Care 8: 594-599

22. Capuzzo M, Valentin A, Alvisi R (2009) Open versus closed units. In: Chice J-D, Moreno R, Putensen C, Rhodes A (eds) Patient Safety and Quality of Care In Intensive Care Medicine. Medizinisch Wissenschaftiche Verlagsgesellschaft, Berlin, pp 177-188

23. Pronovost P, Angus DC, Dorman T, Robinson KA, Dremsizov TT, Young TL (2002) Physician staffing patterns and clinical outcomes in critically ill patients. a systematic review. JAMA 288: $2151-2162$

24. Joynt GM, Gomersall CD, Tan PSK, Lee A, Cheng CAY, Wong ELY (2001) Prospective evaluation of patients refused admission to an intensive care unit: triage, futility and outcome. Intensive Care Med 27: 1459-1465

25. Sprung CL, Geber D, Eidelman LA, et al (1999) Evaluation of triage decisions for intensive care admission. Crit Care Med 27: 1073-1079

26. Task Force on Guidelines, Society of Critical Care Medicine (1999) Recommendations for intensive care unit admission and discharge criteria. Crit Care Med 27: 633-638

27. Garrouste-Orgeas M, Montuclard L, Timsit J-F, et al (2005) Predictors of intensive care unit refusal in French intensive care units: A multiple-center study. Crit Care Med 33: 750-755

28. Lecuyer L, Chevret S, Thiery G, Darmon M, Schlemmer B, Azoulay E (2007) The ICU Trial: A new admission policy for cancer patients requiring mechanical ventilation. Crit Care Med 35: 808-814

29. Azoulay E, Bele N, Thiery G, Schlemmer B (2007) An alternative to refusing ICU admission of cancer patients. In: Kuhlen R, Moreno R, Ranieri M, Rhodes A (eds) 25 Years of Progress and Innovation in Intensive Care Medicine. Medizinisch Wissenschaftliche Verlagsgesellschaft, Berlin, pp 449-458

30. Azoulay E, Metnitz B, Sprung C-L, et al (2009) End-of-life practices in 282 intensive care units: data from the SAPS 3 database. Intensive Care Med 35: 623-630

31. Sprung CL, Woodcock T, Sjokvist P, et al (2008) Reasons, considerations, difficulties and documentation of end-of-life decisions in European intensive care units: the ETHICUS Study. Intensive Care Med 34: 271-277

32. Bülow H-H, Sprung C-L, Reinhart K, et al (2008) The world's major religions' points of view on end-of-life decisions in the intensive care unit. Intensive Care Med 34: 423-430

33. Randall Curtis J (2007) End-of-life care for patients in the intensive care unit. In: Kuhlen R, Moreno R, Ranieri M, Rhodes A (eds) 25 Years of Progress and Innovation in Intensive Care Medicine. Medizinisch Wissenschaftliche Verlagsgesellschaft, Berlin, pp 469-479

34. Tsang KW, Ho PL, Ooi GC, et al (2003) A cluster of cases of severe acute respiratory syndrome in Hong Kong. N Engl J Med 348: 1977-1985

35. Skowronski DM, Petric M, Daly P, et al (2006) Coordinated response to SARS, Vancouver, Canada. Emerg Infect Dis 12: 55-58

36. Rello J, Rodriguez A, Ibanez P, et al (2009) Intensive care adult patients with severe respiratory failure caused by Influenza A (H1N1) in Spain. Crit Care 13: R148

37. Webb SAR, Seppelt IM, for the ANZIC Influenza Investigators (2009) Pandemic (H1N1) 2009 influenza ("swine flu") in Australian and New Zealand intensive care. Crit Care Resusc 11: 170-172 
38. Eastman N, Philips B, Rhodes A (2010) Triaging for adult critical care in the event of overwhelming need. Intensive Care Med 36: 1076-1082

39. Sprung CL, Zimmerman JL, Christian MD, et al (2010) Recommendations for intensive care unit and hospital preparations for an influenza epidemic or mass disaster: summary report of the European Society of Intensive Care Medicine's Task Force for intensive care unit triage during an influenza epidemic or mass disaster. Intensive Care Med 36: $428-443$

40. Metnitz PG, Moreno RP, Almeida E, et al (2005) SAPS 3. From evaluation of the patient to evaluation of the intensive care unit. Part 1: Objectives, methods and cohort description. Intensive Care Med 31: 1336-1344

41. Rosenberg AL, Hofer TP, Hayward RA, Strachan C, Watts CM (2001) Who bounces back? Physiologic and other predictors of intensive care readmission. Crit Care Med 29: $511-518$

42. Hasan M (2001) Readmission of patients to hospital: still ill defined and poorly understood. Int J Qual Health Care 13: 177 - 179

43. Rubins HB, Moskowitz MA (1988) Discharge decision-making in a medical intensive care unit. Identifying patients at high risk of unexpected death or unit readmission. Am J Med 84: $863-869$

44. Bhatia N, Gombar S, Gombar KK, Thapa D (2010) Why patients are readmitted to ICU. J Anaesthesiol Clin Pharmacol 26: 91-93

45. Metnitz PG, Fieux F, Jordan B, Lang T, Moreno R, Le Gall JR (2003) Critically ill patients readmitted to intensive care units--lessons to learn? Intensive Care Med 29: 241-248

46. Halpern NA, Pastores SM (2010) Critical care medicine in the United States 2000-2005: An analysis of bed numbers, occupancy rates, payer mix, and costs. Crit Care Med 38: $65-71$

47. Csomós A, Janecsko M, Edbrooke D (2005) Comparative costing analysis of intensive care services between Hungary and United Kingdom. Intensive Care Med 31: 1280-1283

48. Edbrooke DL, Ridley S, Hibbert CL, Corcoran M (2001) Variations in expenditure between adult general intensive care units in the UK. Anaesthesia 56: 208-216

49. Prien T, Groll O, Geldner G, et al (2002) Ist-Kosten Intensivmedizin deutscher Anasthesieabteilungen-Bezugsjahr 1999. Anasthesiol Intensivmed 43: 244-254

50. Haller G, Stoelwinder J, Myles PS, McNeil J (2009) Quality and safety indicators in anesthesia. A systematic review. Anesthesiology 110: 1158-1175

51. Moreno RP, Rhodes A, Donchin Y (2009) Patient safety in intensive care medicine: the Declaration of Vienna. Intensive Care Med 35: 1667-1672

52. Chakravarthy M (2010) Errors in cardiac anesthesia - A deterrent to patient safety. Ann Cardiac Anaesth 13: 87-88

53. Donchin Y, Gopher D, Olin M, et al (1995) A look into the nature and causes of human errors in the intensive care unit. Crit Care Med 23: 294-300

54. Valentin A, Capuzzo M, Guidet B, et al (2006) Patient safety in intensive care: results from the multinational Sentinel Events Evaluation (SEE) study. Intensive Care Med 32: $1591-1598$

55. Valentin A, Capuzzo M, Guidet B, et al (2009) Errors in the administration of parenteral drugs - an urgent safety issue in intensive care units. Results from a multinational, prospective study. BMJ 338: b814

56. Beydon L, Ledenmat PY, Soltner C, et al (2010) Adverse events with medical devices in anesthesia and intensive care unit patients recorded in the French Safety Database in 2005-2006. Anesthesiology 112: 364-372

57. Zohar D, Livne Y, Tenne-Gazit O, Admi H, Donchin Y (2007) Healthcare climate: A framework for measuring and improving patient safety. Crit Care Med 35: 1312-1317

58. Wiegmann DA, Dunn WF (2010) Changing culture. A new view of human error and patient safety. Chest 137: 250-252

59. Livne Y, Donchin Y (2009) Building a safety culture within the ICU. In: Chiche J-D, Moreno R, Putensen C, Rhodes A (eds) Patient Safety and Quality of Care in Intensive Care Medicine. Medizinisch Wissenschaftiche Verlagsgesellschaft, Berlin, pp 39-46

60. O'Connor MF (2007) Safety culture: Easy to advocate, difficult to create. Crit Care Med 35: 1429 
61. Sexton JB, Helmreich RL, Neilands TB, et al (2006) The Safety Attitudes Questionnaire: psychometric properties, benchmarking data, and emerging research. BMC Health Serv Res 6: 44

62. Ferdinande P, Members of the Task Force of the European Society of Intensive Care Medicine (1997) Recommendations on minimal requirements for intensive care departments. Intensive Care Med 23: 226-232

63. Poole D, Rossi C, Anghileri A, et al (2009) External validation of the Simplified Acute Physiology Score (SAPS) 3 in a cohort of 28,357 patients from 147 Italian intensive care units. Intensive Care Med 35: 1916-1924

64. Poole D, Bertolini G (2009) Outcome-based benchmarking in the ICU Part I: Use and limitations of severity scores in critical care. In: Chiche J-D, Moreno R, Putensen C, Rhodes A (eds) Patient Safety and Quality of Care in Intensive Care Medicine. Medizinisch Wissenschaftiche Verlagsgesellschaft, Berlin, pp 151-160

65. Aujesky D, Mor MK, Geng M, Fine MJ, Renaud B, Ibrahim SA (2008) Hospital volume and patient outcomes in pulmonary embolism. Can Med Assoc J 178: 27-33

66. Lindenauer PK, Behal R, Murray CK, Nsa W, Houck PM, Bratzler DW (2006) Volume, quality of care, and outcome in pneumonia. Ann Intern Med 144: 262-269

67. Glance LG, Dick AW, Osler TM, Mukamel DB (2005) The relation between surgeon volume and outcome following off-pump vs on-pump coronary artery bypass graft surgery. Chest 128: $829-837$

68. Kahn JM (2009) Volume and outcome in intensive care. In: Chice J-D, Moreno R, Putensen C, Rhodes A (eds) Patient Safety and Quality of Care In Intensive Care Medicine. Medizinisch Wissenschaftiche Verlagsgesellschaft, Berlin, pp 161-170

69. Matti R, Sari K, Tero V, et al (2009) Are small hospitals with small intensive care units able to treat patients with severe sepsis? Intensive Care Med 36: 673-679

70. Minvielle E, Aegerter P, Dervaux B, et al (2008) Assessing organizational performance in intensive care units: A French experience. J Crit Care 23: 236-244

71. Eichacker PQ, Natanson C, Danner RL (2007) Separating practice guidelines from pharmaceutical marketing. Crit Care Med 35: 2877-2878

72. Eichacker PQ, Natanson C, Danner RL (2006) Surviving Sepsis - Practice Guidelines, Marketing Campaigns, and Eli Lilly. N Engl J Med 355: 1640-1642

73. Vincent JL, Singer M, Marini JJ, et al (2010) Thirty years of critical care medicine. Crit Care 14: 311

74. Pronovost PJ, Berenholtz SM, Goeschel C, et al (2008) Improving patient safety in intensive care units in Michigan. J Crit Care 23: 207-221

75. Winters BD, Gurses AP, Lehmann H, Sexton JB, Rampersad CJ, Pronovost PJ (2009) Clinical review: Checklists - translating evidence into practice. Crit Care 13: 210

76. Bertolini G, Boffelli, Malacarne S, et al (2010) End-of-life decision-making and quality of ICU performance: an observational study in 84 Italian units. Intensive Care Med 36: $1495-1504$

77. Fassier T, Darmon M, Laplace C, et al (2007) One-day quantitative cross-sectional study of family information time in 90 intensive care units in France. Crit Care Med 35: $177-183$

78. Siegel MD (2009) Lost in translation: Family conferences for families that do not speak English. Crit Care Med 37: 340-341

79. Azoulay E, Pochard F, Chevret S, et al (2001) Meeting the Needs of Intensive Care Unit Patient Families. A Multicenter Study. Am J Respir Crit Care Med 163: 135-139

80. Goodman DC, Fisher ES (2008) Physician Workforce Crisis? Wrong Diagnosis, Wrong Prescription. N Engl J Med 358: 1658-1661

81. Samb B, Celletti F, Holloway J, Van Damme W, De Cock KM, Dybul M (2007) Rapid expansion of the health workforce in response to the HIV epidemic. N Engl J Med 357: $2510-2514$

82. Angus DC, Kelley MA, Schmitz RJ, White A, Popovich J, for the Committee in Manpower for Pulmonary and Critical Care Societies (COMPACCS) (2000) Caring for the critically ill patient. Current and projected workforce requirements for care of the critically ill and patients with pulmonary disease: can we meet the requirements of an aging population? JAMA 284: $2762-2770$ 
83. Sapirstein A, Lone N, Latif A, Fackler J, Pronovost PJ (2009) Tele ICU: paradox or panacea? Best Pract Res Clin Anaesthesiol 23: 115-126

84. Yoo EJ, Dedley RA (2009) Evaluating telemedicine in the ICU. JAMA 302: 2705-2706

85. Thomas EJ, Lucke JF, Wueste L, Weavind L, Patel B (2009) Association of telemedicine for remote monitoring of intensive care patients with mortality, complications, and length of stay. JAMA 302: 2671-2678

86. Bion J, Reay H, Bullock A (2007) Training in intensive care medicine. In: Kuhlen R, Moreno R, Ranieri M, Rhodes A (eds) 25 Years of Progress and Innovation in Intensive Care Medicine. Medizinisch Wissenschaftliche Verlagsgesellschaft, Berlin, pp 351-358

87. Perkins GD, Barrett H, Bullock I, et al (2005) The Acute Care Undergraduate TEaching (ACUTE) Initiative: consensus development of core competencies in acute care for undergraduates in the United Kingdom. Intensive Care Med 31: 1627-1633

88. The CoBaTrICE Collaboration (2009) The educational environment for training in intensive care medicine: structures, processes, outcomes and challenges in the European region. Intensive Care Med 35: 1575-1583

89. Park CS, Rochlen LR, Yaghmour E, et al (2010) Acquisition of critical intraoperative event management skills in novice anesthesiology residents by using high-fidelity simulationbased training. Anesthesiology 112: 202-211

90. Cooper JB, Murray D (2010) Simulation training and assessment. a more efficient method to develop expertise than apprenticeship. Anesthesiology 112: 8-9

91. Pardo M Jr (2010) Anesthesia: How to organize and train our teachers. Anesthesiology 112: $773-774$

92. McMahon GT, Katz JT, Thorndike ME, Levy BD, Loscalzo J (2010) Evaluation of a redesign initiative in an internal-medicine residency. N Engl J Med 362: 1304-1311 